\title{
Risk Factors for Recurrent Pulmonary Exacerbation in Idiopathic Pulmonary Hemosiderosis
}

\author{
Mina Gharibzadeh Hızal ${ }^{1}$, Sanem Eryılmaz Polat ${ }^{2}$, Tugba Ramaslı Gursoy ${ }^{3}$, beste \\ Ozsezen $^{4}$, Dilber Ademhan Tural ${ }^{5}$, Jale Karakaya ${ }^{2}$, Nagehan Emiralioğlu ${ }^{2}$, Sevgi Pekcan ${ }^{6}$, \\ Ayşe Aslan ${ }^{3}$, Ebru Yalcin ${ }^{5}$, Deniz Dogru², ugur ozcelik², and Nural Kiper ${ }^{2}$ \\ ${ }^{1}$ hacettepe university \\ ${ }^{2}$ Hacettepe University \\ ${ }^{3}$ Gazi Universitesi Tip Fakultesi \\ ${ }^{4}$ Hacettepe Universitesi Tip Fakultesi \\ ${ }^{5}$ Hacettepe University Faculty of Medicine \\ ${ }^{6}$ Meram Medical Faculty
}

July 7,2020

\begin{abstract}
Objectives: To evaluate the risk factors of recurrent pulmonary exacerbation and poor prognosis in children with idiopathic pulmonary hemosiderosis (IPH). Methods: In this multicenter study, 54 patinets with diagnosis of IPH included. Medical records were retrospectively reviewed from three tertiary care hospitals between 1979 and 2019. Also, current information and the long-term progress of patients was determined by contacting the families by telephone. Results: A total of 54 children were included. The median age of onset of symptoms was $4.5 \pm 3.8$ years. The median time from onset to diagnosis was 0.9 years \pm 2.2 . The mean number of recurrent episodes per child in the recurrence-positive group was 3.55 (1-15). Univariate analysis demonstrated that patients presenting with hypoxia or requiring transfusion at the time of presentation had significantly more recurrence episodes $(\mathrm{P}=0.002)$. Multivariate analysis showed that the presence of hypoxia at the time of initial presentation was a significant independent predictor of recurrent episodes $(\mathrm{P}=0.027)$. The median follow-up was $3.3 \pm 4.8$ years $(0.75$ months-27 years). There was a significant relationship between the presence of hypoxia, transfusion history, ANA positivity, and elevated transaminases at the time of initial evaluation and treatment response. Conclusions: The present study provides important information on the clinical course and outcome of pediatric IPH, and substantial information regarding factors that affect recurrent exacerbations and prognosis. Demonstrating of hypoxia as an independent risk factor in recurrence episodes could be guide physicians in the planning of treatment strategies.
\end{abstract}

\section{Introduction}

Idiopathic pulmonary hemosiderosis (IPH) is one of the uncommon reasons for diffuse alveolar hemorrhage, with an estimated incidence of 0.24-1.23 per million. ${ }^{1-3}$ The definition of IPH is used in cases where the underlying cause of the pulmonary bleeding cannot be identified with extensive investigations. ${ }^{3,4}$ The disease is manifested by pulmonary hemorrhage and characterized by the triad of iron deficiency anemia, hemoptysis, and pulmonary infiltrates on chest imaging ${ }^{5-9}$. Although the pathophysiology of the disease unknown, different hypotheses have been proposed regarding the underlying mechanism of the disorder such as environmental, auto-immune, allergic, genetic. ${ }^{10,11}$ The course of the disease is highly variable from single to recurrent exacerbations. Some patients recover fully, whereas others develop pulmonary fibrosis and respiratory insufficiency. ${ }^{12-14}$

Currently, there is a lack of consensus regarding the management of IPH. ${ }^{12,15}$ There is limited information 
about factors that can help in predicting patients with recurrent exacerbations at the time of diagnosis. There is also no consensus regarding the definition of pulmonary exacerbations for children with IPH.

The purpose of this study was to investigate the clinical characteristics and prognosis of patients with IPH, and to evaluate the incidence and risk factors of recurrent pulmonary exacerbations. Also, we tried to investigate factors that contributed to treatment responses.

\section{Method}

All the children who were diagnosed as having IPH (regardless their clinical severity) and followed up in three tertiary care hospitals between 1979 and 2019 (Hacettepe University Medical Faculty Children's Hospital, Gazi University Medical Faculty Children's Hospital, Meram University Medical Faculty Children's Hospital) and whose records were accessed included in the study. Data were collected from medical records retrospectively. Also, current information and the long-term progress of patients was determined by contacting the families by telephone. Ethics clearance was obtained from the Institute Ethics Committee. Criteria for diagnosis were based on iron deficiency anemia without any other apparent cause, respiratory symptoms (including hemoptysis, cough, and dyspnea), and pulmonary parenchymal infiltrates on chest imaging and exclusion of other diseases. Diagnosis was confirmed by the presence of hemosiderin-laden macrophages in bronchoalveolar lavage (BAL), gastric aspirate and/or in lung tissue specimens. The diagnosis, treatment, and follow-up of patients was performed according to the IPH management protocol of the units.

Clinical information was collected from medical records, including demographics, clinical features, comorbidities, personal history, environmental exposure, and pulmonary function testing (PFT). Hypoxia was defined by presence of oxygen saturation below $93 \%$. The reviewed laboratory data included hemoglobin, leukocyte and reticulocyte counts, total immunoglobulin levels, and autoimmune markers. Skin tests and specific allergen statuses were recorded. Chest imaging, including X-ray and chest tomography, was evaluated from both reports and imaging. Detailed treatment information recorded.

Clinical recurrence was defined as new-emerging pulmonary infiltration in combination with acute clinically significant deterioration of respiratory symptoms and/or a decline in PFT plus new-onset hemoptysis and/or anemia after a symptom-free period of at least two months, which eventually necessitated a change in regular management. Non-recurrence was defined as resolution of clinical symptoms and imaging abnormalities, or clinical finding improvement with retention or stability of imaging abnormalities when treatment was withdrawn or who died after first attack. Patients without recurrence after the initial treatment and with complete resolution of findings were classified as having a good response. Patients who had a recurrence but responded well to oral steroids, or with reduced need for transfusion, or with minimal residual findings were classified as having a partial response. Patients who did not respond to steroids and required further immunosuppressant agents or died were classified as poor response.

The normality of distribution for numerical variables was checked using the Kolmogorov-Smirnov test. Descriptive statistics were expressed as mean \pm standard deviation or median (min-max) according to the assumption of normal distribution. When data were not normally distributed, the Mann-Whitney U test was used to compare the differences between two independent groups for quantitative variables. The KruskalWallis test was used to compare the difference between three or more than groups in non-normal quantitative variables. After the Kruskal-Wallis test, the Dunn test was used as a post hoc test. Fisher's exact tests were used to examine the difference between groups for categorical variables. Binary logistic regression with the backward method was used to evaluate which independent variables (oxygen saturation $<93$, presence of hemoptysis at the beginning, age of initiation of symptoms, hepatomegaly) were statistically significant predictors of recurrent exacerbations. Odds ratios with $95 \%$ confidence intervals (CIs) were calculated for potential predictors of risk factors for recurrent exacerbations. A $\mathrm{p}<0.05$ was accepted as statistically significant. Data were analyzed using the IBM SPSS statistics 23.0 software package.

\section{Results}

A total of 54 children included in the study. The median time from onset to diagnosis was 0.9 years \pm 2.2 
(0.25 months to 12 years). Consanguinity (first/second/third degree cousin) between parents was present in 26 patients. The brother of one patient died after an episode of similar symptoms. The main initial manifestations of the patients at diagnosis were as follows: cough $(n=41,75.9 \%)$, dyspnea $(n=29,53.7 \%)$, hemoptysis $(\mathrm{n}=29,53.7 \%)$. The classic triad of anemia, hemoptysis, and pulmonary infiltration in imaging was found in $24(47.1 \%)$. While hemoptysis was initially observed in $29(53.7 \%)$ patients, it's determined in $33(61.1 \%)$ patients, including follow-up time. A total of $41(75.9 \%)$ patients were initially misdiagnosed; the most common misdiagnosis was iron deficiency anemia and pneumonia. The main demographic, clinical, laboratory, and radiologic findings at diagnosis are described in Table 1 and Table 2.

Records of hemosiderin-laden macrophages in BAL were found for 24 patients, and all were positive. Gastric aspirate from 42 patients was examined for hemosiderin-laden macrophages, and $28(66.6 \%)$ patients were positive. Six patients had negative results in gastric aspirate, although their hemosiderin-laden macrophages in BAL were positive. BAL fluid was positive for the following microorganisms in eight patients: coronavirus $(n=2)$, parainfluenza type $1(n=2)$, respiratory syncytial virus, cytomegalovirus $(n=1)$, Haemophilus influenzae $(n=2)$, and Klebsiella pneumonia $(n=1)$. Five children underwent a lung biopsy, which documented features of pulmonary hemosiderosis with no sign of capillaritis. Thirty-seven patients received blood transfusions. Seven patients also needed transfusions after diagnosis.

Thirty-seven patients with complete current information of recurrence (as provided in telephone conversations) were further analyzed According to the presence of recurrent episodes, patients were dichotomized into recurrence-positive and recurrence-negative groups. The mean number of recurrent episodes per child in the recurrence-positive group was 3.55 (1-15). As seen in Table 3, univariate analysis demonstrated that the recurrence-positive group presented more frequently with hypoxia $(\mathrm{P}=0.002)$. Patients with a history of transfusion before or at the time of the initial diagnosis had significantly higher recurrence rates $(\mathrm{P}=0.002)$. Multivariate regression analysis showed that the presence of hypoxia at the time of initial presentation was a significant independent predictor of recurrent episodes $(\mathrm{P}=0.027)$. Ten patients reported that their recurrence attacks were triggered after upper respiratory infections. Two patients reported a decrease in attack frequency after the influenza vaccine. However, no seasonal relationship was determined with recurrence attacks.

Immunoglobulin E level was elevated in six patients. Cow milk allergy was evaluated in 36 patients with one or more of the following methods: skin tests, radioallergosorbent test (RAST) for milk protein, allergenspecific immunoglobulin E, and provocation test; five of whom were allergic to milk. Two patients were considered as having Heiner syndrome in the follow-up period. At least one antibody was positive in 19 of 54 patients. Autoimmune screening results are given in Table 4. During the follow-up period, ANA positivity developed in one patient, and antigliadin positivity disappeared in one other patient.

Only 16 patients were able to perform the pulmonary function test at the time of admission; 24 patients were aged under five years, and the remainder could not perform the tests due to acute disease conditions. The pulmonary function test was normal in 10/16 patients. Four of them had a restrictive pattern, and two had both restrictive and obstructive patterns.

Among the 54 patients, one patient was diagnosed as having coeliac disease after the initial evaluation. One patient had arthralgia at the time of diagnosis and was diagnosed as having familial Mediterranean fever three years later. One patient developed arthralgia three years later and was diagnosed as having juvenile idiopathic arthritis. One patient had hematuria, but the renal biopsy was normal without specific abnormalities suggestive of Goodpasture syndrome. One patient had a positive result for anti-glomerular basement membrane (GBM), but the renal biopsy was normal. One patient with anti-GBM positivity was diagnosed as having Goodpasture syndrome during follow-up. Coombs-positive hemolytic anemia developed in one patient in the follow-up period. One patient developed end-stage kidney failure due to nephrotic syndrome 20 years after the initial diagnosis while she was in remission for IPH (ANCA result was negative and biopsy not suggestive of Goodpasture syndrome). Pulmonary hypertension was determined in three patients during follow-up. Three patients were evaluated for COPA mutations, and all were negative. 
All children were treated with systemic corticosteroids on initial diagnosis. The mean duration of the total prednisolone course was 22 (range, 0.75-72) months. Hydroxychloroquine treatment was started in eight patients, cyclophosphamide treatment was started in four patients, azathioprine treatment was started in two patients, and inhaled steroids were started in 16 patients during the reduction of steroid treatment doses. In six patients, steroid-related adverse effects were observed. In two patients, treatment was discontinued due to steroid adverse effects. The median recurrence time of attacks after cessation of corticosteroid treatment was 6 (range, 2-30) months. Of the 54 treated patients, seven died of pulmonary hemorrhage and/or respiratory failure.

One patient discontinued steroid treatment after two months and was lost to follow-up. When the patient was contacted again, he stated that he had been depressed and had stopped all treatments because he had gained weight and developed hypertension after steroid treatment. Six years passed since the patient stopped treatment and he had mild hemoptysis every day and shortness of breath with effort. A new CT revealed bilateral patchy infiltration areas with ground-glass appearance.

Thirty-seven patients with complete long-term follow-up and treatment information were further analyzed. Patients were categorized into three groups according to treatment response; good, partial, and poor. Thirtyseven patients with long-term follow-up or current information (as provided in telephone conversations) were included in this grouping. There was no significant difference in terms of the age of diagnosis, delay in diagnosis duration, sex, onset time of symptoms, consanguinity, initial signs, radiologic findings, and PFT. There was a significant relationship between the presence of hypoxia, transfusion history, ANA positivity, and elevated transaminases at the time of initial evaluation and treatment response, as seen in Table 5 and Figure 1.

\section{Discussion}

There is no consensus in terms of the evaluation and management of children with IPH. ${ }^{12,15}$ To the best of our knowledge, this study is one of the largest with a long follow-up period, which demonstrated that the presence of hypoxia at diagnosis could be an independent risk factor for recurrent episodes. Moreover, our study is the first to indicate that prognosis might be poor in patients with ANA positivity at the time of diagnosis.

Only one recent study has attempted to identify predictors for recurrence episodes in patients with IPH. ${ }^{16}$ However, the focus of this study was not to predict patients with recurrence episodes, and only 10 patients with recurrence episodes were evaluated and its follow-up period was shorter than the present study. Their results suggested that bilirubin levels were significantly higher in children with recurrence episodes. The results of our study, on the other hand, revealed that patients presenting with hypoxia or requiring transfusion at the time of diagnosis had significantly more recurrence episodes. Moreover, multivariate regression analysis demonstrated that hypoxia was an independent risk factor in predicting recurrence episodes. To the best of our knowledge, our study is the first to indicate that the presence of hypoxia at initial presentation is an independent risk factor for recurrent IPH episodes. Another significant result of our study was that important factors such as age at the onset of symptoms, length of delay in diagnosis, clinical features, physical examination findings, and laboratory results were not helpful in predicting recurrence episodes. This study provides valuable information to physicians that may guide the management of treatment for patients who present with hypoxia.

IPH may occur at any age but it is more frequently reported in children between the ages of 1 and 7 years, similar to the results of present study. ${ }^{17,18}$ The results of our study revealed that the symptom onset age was 3 months for the youngest, and 15 years of age for the oldest patient. This result demonstrates the fact that IPH can be seen in all age groups and physicians should be careful not to focus on younger patients only.

Consanguinity was present in $48 \%$ of the patients in our study. This rate proves to be more than the double the rate of consanguinity in Turkey $(20 \%) .{ }^{19}$ Furthermore, a sibling one of our patient's died with similar symptoms. Our result, in line with those of previous studies, indicates that genetics might play a role in the underlying mechanisms of IPH. ${ }^{20-23}$ 
Studies in the literature reported varying results regarding the presenting symptoms of the disease ${ }^{24-26}$. In our study, the most common presenting features at onset were anemia, cough, dyspnea, and hemoptysis. In their study, Tayard et al. also reported that anemia and dyspnea were the most common symptoms. ${ }^{20}$ In the study conducted by Zhang et al., however, cough and hemoptysis were the predominant symptoms, and dyspnea was observed in $15 \%$ of the patients. ${ }^{16}$

Although hemoptysis is one of the components of the classic triad of the disease, it only occurs in around half of all patients at initial presentation. ${ }^{16,20}$ Yet it has been reported that hemoptysis was seen in almost all adult patients during the course of the disease. ${ }^{3}$ The results of studies conducted by French and Chinese researchers revealed that hemoptysis was observed in around half of all patients, as was the case in our study. ${ }^{16,20}$ In spite of the fact that opinions suggesting that hemoptysis might go unnoticed due to sputum swallowing in young children, ${ }^{27}$ no evident hemoptysis was observed in $39 \%$ of patients covered by our study with a long follow-up period. It should be remembered that evident hemoptysis might never emerge in a substantial part of patients, including during the course of the disease.

Studies thus far have reported delays in diagnosis to range between 1 and 6.3 years. ${ }^{20,28}$ In our study, evidence of remarkable delay in diagnosis was observed in some patients. Two of the most significant reasons for this situation are the absence of the known classic triad in half of all patients and the low level of awareness of pediatricians about this disease. Further, the three centers in which our study was conducted were reference centers in metropolitans. It takes time for patients, to access such centers, and a significant portion of the patients lose time because they receive treatment having been pre-diagnosed as having iron deficiency anemia and pneumonia. In order to prevent delays in diagnosis, particularly in patients with no classic triad symptoms, awareness about the disease should be increased and physicians should adopt a high level of suspicion to this end.

Multiple theories have been proposed regarding the potential underlying pathophysiology of the disease. ${ }^{7,10,29}$ Even though some evidence has been reported in the literature regarding the relationship between IPH and autoimmune diseases, this association still remains controversial. ${ }^{20,30,31}$ Previous studies speculated that more than one-quarter of patients developed an immune disorder later in follow-up. ${ }^{30}$ At least one antibody was found to be positive in $35 \%$ of patients covered by our study but accompanying autoimmune diseases were observed in very few patients. In long-term follow-ups, however, four patients were observed to have accompanying autoimmune diseases.

It has been strongly suggested that there is a relationship between celiac disease and IPH. Researchers believe that an immunologic pathogenesis underlies this combination, which has yet to be clarified. ${ }^{32-34}$ The results of our study revealed that celiac antibody positivity was $30 \%$, even though only one patient was diagnosed as having celiac disease upon further investigation. Also, celiac antibody positivity disappeared in one other patient. The high celiac autoantibody positivity in our patients increases the possibility that IPH may be within the spectrum of autoimmune diseases. Also, it is important to keep in mind that antibody results could change over time.

Our present study provides support for importance of BAL in diagnosis of IPH; six patients had negative results in gastric aspirate, although their hemosiderin-laden macrophages in BAL were positive. Furthermore, we are not able to evaluate the trigger effects of infectious agents in the exacerbation of IPH because of limited microbiologic records. However, some of our patients clearly stated that attacks were triggered by infection, indicating that further research is required especially to elucidate on the role of influenza in triggering attacks.

Our study also aimed to predict the treatment responses of patients. Transfusion history, presence of hypoxia, ANA positivity, and elevated transaminases enzymes at presentation were associated with poor treatment response. The limited number of studies in the literature investigating the possible factors affecting prognosis at the time of diagnosis yielded different results. A study conducted in Greece in 1983 reported poorer prognoses for young male children. ${ }^{35}$ In another study conducted in Japan in 1995, on the other hand, the authors argued that prognosis might be poorer in patients with fever, leukocytosis, hepatomegaly, and splenomegaly. ${ }^{16}$ In a study conducted in India, the authors associated hemoptysis and high bilirubin at 
diagnosis with poor prognosis. ${ }^{36}$ A previous study of 15 patients indicated that the presence of ANCA was associated with poor prognosis in IPH. ${ }^{30}$ Blanco et al. proposed that ANCA positivity might have been associated with poor prognosis their study conducted with four patients with IPH with no systemic or renal disease ${ }^{37}$ Interestingly, our results revealed that the patient group with ANA positivity had poorer prognoses. To our knowledge, this association has never been reported in the literature. Prognostic value of autoantibodies was evaluated in many studies; presence of ANA was associated with a more severe disease in certain connective tissue disorders ${ }^{38}$. Our result on prognosis evaluation may be random due to the fact that the number of patients per group was limited, but it might also be related to the possibility of accompanying autoimmune events that could have affected prognosis that have yet to be identified in the ANA- positive group. Our study underlined this relationship but in order to clarify this relationship, further larger and prospective studies are needed.

There are no prospective studies on the management of IPH in children for which most of the evidence is delivered from observational studies. ${ }^{12}$ It was seen that the second treatment of choice following steroids was hydroxychloroquine, and inhaler steroid treatment was used in one-third of the patients. It was also observed that steroid treatment was generally well tolerated by the patients and adverse effects that led to steroid discontinuation were quite few. It was, however, seen that the patients often went through relapse episodes during the downgrading or discontinuation of steroid treatment. Such episodes were most commonly observed 6 months following steroid discontinuation, but there was a case of recurrence after 30 months. It is therefore important to closely follow-up patients even if they have no symptoms after steroid discontinuation.

One of the main limitations of our study is its retrospective design. However, the study data were enhanced by contacting the patients to collect their current data and long-term prognoses. Another limitation was that patients from different centers were included in the study and there were interdepartmental differences in follow-up protocols. Further, there have been changes in examinations in patient assessment and treatment management because the study covered a period of 40 years.

In conclusion, our study offers significant results to the literature because it has a large population and covers a long period of follow-up. The results of our study demonstrated that the presence of hypoxia at diagnosis could be an independent risk factor for recurrent episodes. This information may guide physicians in the planning treatment strategies for patients who present with hypoxia. Further, our study is the first to indicate that prognosis might be poor in patients with ANA positivity at the time of diagnosis but this result needs to be clarified by further studies.

\section{References}

1. Bakalli I, Kota L, Sala D, et al. Idiopathic pulmonary hemosiderosis - a diagnostic challenge. Ital J Pediatr. 2014;40:35.

2. Kjellman B, Elinder G, Garwicz S, Svan H. Idiopathic pulmonary haemosiderosis in Swedish children. Acta Paediatr Scand.1984;73(5):584-588.

3. Ioachimescu OC, Sieber S, Kotch A. Idiopathic pulmonary haemosiderosis revisited. Eur Respir J. 2004;24(1):162-170.

4. Zhang X, Wang L, Lu A, Zhang M. Clinical study of 28 cases of paediatric idiopathic pulmonary haemosiderosis. J Trop Pediatr.2010;56(6):386-390.

5. Kiper N, Gocmen A, Ozcelik U, Dilber E, Anadol D. Long-term clinical course of patients with idiopathic pulmonary hemosiderosis (1979-1994): prolonged survival with low-dose corticosteroid therapy. Pediatr Pulmonol. 1999;27(3):180-184.

6. Kamienska E, Urasinski T, Gawlikowska-Sroka A, Glura B, Pogorzelski A. Idiopathic pulmonary hemosiderosis in a 9-year-old girl. Eur J Med Res. 2009;14 Suppl 4:112-115.

7. AlJassmi AM. Autoimmunity and Delayed Diagnosis in Pediatric Idiopathic Pulmonary Hemosiderosis. $J$ Pediatr Hematol Oncol.2019. 
8. Bhatia S, Tullu MS, Vaideeswar P, Lahiri KR. Idiopathic pulmonary hemosiderosis: alveoli are an answer to anemia. J Postgrad Med.2011;57(1):57-60.

9. Pedersen FM, Milman N. [Idiopathic pulmonary hemosiderosis]. Ugeskr Laeger. 1996;158(7):902-904.

10. Moissidis I, Chaidaroon D, Vichyanond P, Bahna SL. Milk-induced pulmonary disease in infants (Heiner syndrome). Pediatr Allergy Immunol. 2005;16(6):545-552.

11. Corrin B, Jagusch M, Dewar A, et al. Fine structural changes in idiopathic pulmonary haemosiderosis. J Pathol.1987;153(3):249-256.

12. Chin CI, Kohn SL, Keens TG, Margetis MF, Kato RM. A physician survey reveals differences in management of idiopathic pulmonary hemosiderosis. Orphanet J Rare Dis. 2015;10:98.

13. Alimi A, Taytard J, Abou Taam R, et al. Pulmonary hemosiderosis in children with Down syndrome: a national experience. Orphanet $J$ Rare Dis. 2018;13(1):60.

14. Saeed MM, Woo MS, MacLaughlin EF, Margetis MF, Keens TG. Prognosis in pediatric idiopathic pulmonary hemosiderosis. Chest.1999;116(3):721-725.

15. Kurland G, Deterding RR, Hagood JS, et al. An official American Thoracic Society clinical practice guideline: classification, evaluation, and management of childhood interstitial lung disease in infancy. Am $J$ Respir Crit Care Med. 2013;188(3):376-394.

16. Zhang Y, Luo F, Wang N, Song Y, Tao Y. Clinical characteristics and prognosis of idiopathic pulmonary hemosiderosis in pediatric patients. J Int Med Res. 2019;47(1):293-302.

17. Ohga S, Takahashi K, Miyazaki S, Kato H, Ueda K. Idiopathic pulmonary haemosiderosis in Japan: 39 possible cases from a survey questionnaire. Eur J Pediatr. 1995;154(12):994-995.

18. Morgan PG, Turner-Warwick M. Pulmonary haemosiderosis and pulmonary haemorrhage. Br J Dis Chest. 1981;75(3):225-242.

19. Pinar G, Kaplan S, Pinar T, et al. The prevalence and risk factors for osteoporosis among 18- to 49-yearold Turkish women. Women Health. 2017;57(9):1080-1097.

20. Taytard J, Nathan N, de Blic J, et al. New insights into pediatric idiopathic pulmonary hemosiderosis: the French RespiRare (R) cohort. Orphanet Journal of Rare Diseases. 2013;8.

21. Matsaniotis N, Karpouzas J, Apostolopoulou E, Messaritakis J. Idiopathic pulmonary haemosiderosis in children. Arch Dis Child.1968;43(229):307-309.

22. Beckerman RC, Taussig LM, Pinnas JL. Familial idiopathic pulmonary hemosiderosis. Am J Dis Child. 1979;133(6):609-611.

23. Gencer M, Ceylan E, Bitiren M, Koc A. Two sisters with idiopathic pulmonary hemosiderosis. Can Respir J. 2007;14(8):490-493.

24. Willms H, Gutjahr K, Juergens UR, et al. [Diagnostics and therapy of idiopathic pulmonary hemosiderosis]. Med Klin (Munich).2007;102(6):445-450.

25. Poggi V, Lo Vecchio A, Menna F, Menna G. Idiopathic pulmonary hemosiderosis: a rare cause of irondeficiency anemia in childhood.J Pediatr Hematol Oncol. 2011;33(4):e160-162.

26. Afzal N, Mushtaq A, Rahman A, Qureshi S. Idiopathic pulmonary haemosiderosis presenting as severe iron deficiency anaemia-a case from Pakistan. J Pak Med Assoc. 2012;62(8):845-847.

27. de Silva C, Mukherjee A, Jat KR, Lodha R, Kabra SK. Pulmonary Hemorrhage in Children: Etiology, Clinical Profile and Outcome.Indian J Pediatr. 2019;86(1):7-11. 
28. Luo XQ, Ke ZY, Huang LB, et al. Maintenance therapy with dose-adjusted 6-mercaptopurine in idiopathic pulmonary hemosiderosis.Pediatr Pulmonol. 2008;43(11):1067-1071.

29. Reading R, Watson JG, Platt JW, Bird AG. Pulmonary haemosiderosis and gluten. Arch Dis Child. 1987;62(5):513-515.

30. Le Clainche L, Le Bourgeois M, Fauroux B, et al. Long-term outcome of idiopathic pulmonary hemosiderosis in children. Medicine (Baltimore). 2000;79(5):318-326.

31. Lemley DE, Katz P. Rheumatoid-like arthritis presenting as idiopathic pulmonary hemosiderosis: a report and review of the literature. $J$ Rheumatol. 1986;13(5):954-957.

32. Berger N, Nichols J, Datta D. Idiopathic pulmonary haemosiderosis with celiac disease (Lane-Hamilton syndrome) in an adult - a case report. Clin Respir J. 2016;10(5):661-665.

33. Lane DJ, Hamilton WS. Idiopathic steatorrhoea and idiopathic pulmonary haemosiderosis. $B r$ Med $J$. 1971;2(5753):89-90.

34. Sethi GR, Singhal KK, Puri AS, Mantan M. Benefit of gluten-free diet in idiopathic pulmonary hemosiderosis in association with celiac disease. Pediatr Pulmonol. 2011;46(3):302-305.

35. Chryssanthopoulos C, Cassimos C, Panagiotidou C. Prognostic criteria in idiopathic pulmonary hemosiderosis in children. Eur J Pediatr.1983;140(2):123-125.

36. Kabra SK, Bhargava S, Lodha R, Satyavani A, Walia M. Idiopathic pulmonary hemosiderosis: clinical profile and follow up of 26 children.Indian Pediatr. 2007;44(5):333-338.

37. Blanco A, Solis P, Gomez S, Valbuena C, Telleria JJ. Antineutrophil cytoplasmic antibodies (ANCA) in idiopathic pulmonary hemosiderosis.Pediatr Allergy Immunol. 1994;5(4):235-239.

38. Didier K, Bolko L, Giusti D, et al. Autoantibodies Associated With Connective Tissue Diseases: What Meaning for Clinicians? Front Immunol. 2018;9:541.

\section{Hosted file}

IPH manuscript Table Pediatric Pulmonology.docx available at https://authorea.com/users/ 337441/articles/463576-risk-factors-for-recurrent-pulmonary-exacerbation-in-idiopathicpulmonary-hemosiderosis 
I

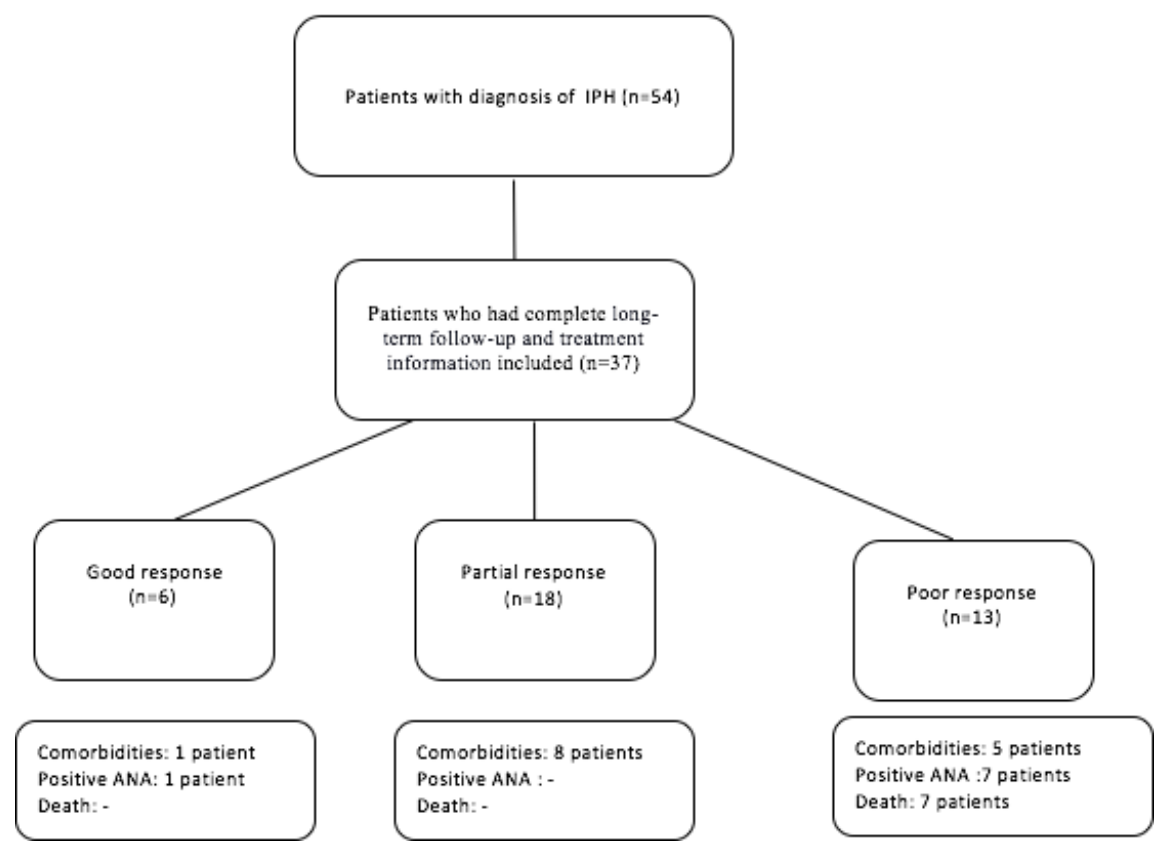

